\title{
Dossiê
}

\section{INOVAÇÃO TECNOCIENTÍFICA E AS RESPONSABILIDADES DE CIENTISTAS E CIENTISTAS SOCIAIS ${ }^{1}$}

Hugh Lacey

Swarthmore College e USP / Associação Filosófica 'Scientiae Studia'

\begin{abstract}
RESUMO
Nas décadas recentes, aumentaram as pressões para dar prioridade à pesquisa que visa gerar inovações tecnocientíficas que prometam contribuir o fortalecimento do crescimento econômico. Neste artigo, pretendo explorar algumas ambiguidades científicas e éticas que têm origem nessas pressões, bem como seus impactos e, à luz das mesmas, algumas responsabilidades incorridas por cientistas e cientistas sociais. Primeiro, vou discutir alguns aspectos da tecnociência e sua relação com a tradição científica moderna. A partir desta discussão, vou fazer três propostas sobre as responsabilidades que cientistas incorrem coletivamente como profissionais em instituições de pesquisa e como membros de organizações científicas. 2 Finalmente, indicarei como o exercício destas responsabilidades depende de contribuições das ciências sociais. Meu argumento detém-se no fato de que, geralmente, assuntos ligados à metodologia, às prioridades e resultados da pesquisa, e à ética estão imbricados nas atividades científicas, e que as responsabilidades éticas dos cientistas não podem ser separadas das suas responsabilidades para manter a integridade da ciência.
\end{abstract}

Palavras-chave: Estudos sociais da ciência, tecnociência, atividade científica, ética

\begin{abstract}
In the last decades the pressure to give priority to research that aims to generate technoscientific innovations has increased. Its main promise is to contribute to economic growth. In this article, we explore some scientific and ethical ambiguities arising from these pressures, in addition to its impacts. In considering this scenario, I seek also to identify some responsibilities involving scientists and social scientists . First, I will discuss some aspects of techno-science and its relationship to modern scientific tradition. From this discussion, I will make three proposals on the responsibilities that scientists collectively incur as professionals in research institutions and as members of scientific organizations. Finally, I will indicate how the exercise of these responsibilities depends on contributions from the social sciences. My argument holds up that, generally, issues related to methodology, priorities and results of the research, and ethics are intertwined in scientific activities, and that the ethical responsibilities of scientists can not be separated from their responsibilities to maintain integrity of science.
\end{abstract}

Key words: Social science studies, techno-science, scientific activity, ethics.

\footnotetext{
${ }^{1}$ Conferência, III EICS, Pelotas, RS, 8 de Outubro de 2012. Quero agradecer ao Prof. Léo Peixoto Rodrigues pala a sua revisão cuidadosa do artigo.

${ }^{2}$ Não discutirei as responsabilidades que cientistas assumam individualmente ou devem assumir na sua pesquisa, ou aquelas que se compartilham igualmente com outras cidadãos, ou questões éticas que devem ser consideradas em pesquisa experimental conduzida responsavelmente.
} 


\section{TECNOCIÊNCIA: METODOLOGIAS DA CIÊNCIA MODERNA E PROGRESSO TECNOLÓGICO}

\section{Metodologia da ciência moderna e progresso tecnológico}

Os intérpretes da ciência moderna sempre (embora de maneiras diferentes) vincularam a descoberta do conhecimento científico a poderes humanos para exercer controle sobre objetos naturais e assim ao progresso tecnológico (MARICONDA, 2006). Aumentar os poderes humanos do controle foi, para Bacon, a finalidade da ciência e teste último do conhecimento científico; para Galileu, fonte do financiamento; para Descartes e Newton, consequência bem vinda de obter conhecimento científico. Além disso, ao longo da tradição, invenções tecnológicas tornaram-se sempre mais essenciais para construir instrumentos e aparelhos usados em pesquisa experimental e observacional; e, às vezes, nas próprias invenções tecnologias estavam os objetos imediatos da investigação científica. Então, o empreendimento da ciência moderna e busca do progresso tecnológico reforçavam-se mutuamente (LACEY, 2008a; 2010). Reflete que o tipo de conhecimento normalmente procurado na ciência moderna - o da ordem causal subjacente a coisas e aos fenômenos e suas leis - serve para informar e motivar a invenção, construção e funcionamento dos objetos tecnológicos (tecnocientíficos).

Este conhecimento normalmente está formulado e verificado dentro de teorias que postulam representações das estruturas subjacentes de coisas e fenômenos, dos processos e interações dos seus componentes, e das leis que os governam. Estas teorias encapsulam possibilidades abertas às coisas e fenômenos em virtude do poder generativo da ordem causal subjacente (governado por leis), inclusive de possibilidades para exercer controle sobre objetos naturais. Nelas, coisas e fenômenos são representados em dissociação dos seus contextos humanos e sociais - de qualquer lugar que tenham no domínio da experiência humana e de seu valor; de qualquer relação com arranjos sociais e vidas humanas; de qualquer ligação com agência humana, valor e qualidades sensórias; e de quaisquer possibilidades que possam ganhar em virtude dos seus lugares em contextos sociais, humanos e ecológicos.

Assim, muito das categorias, que é costumeiro utilizar na vida diária para fins de descrição, comunicação e deliberação (por exemplo, as categorias intencionais e aquelas que designam as qualidades sensoriais) não são utilizadas nessas teorias. Os dados empíricos, aos 


\section{Inovação Tecnocientífica e as responsabilidades de cientistas e Cientistas Sociais}

quais estas teorias devem ajustar-se, também não usam essas categorias - são obtidos a partir da observação dos fenômenos representados nas teorias, tipicamente fenômenos criados em práticas experimentais ou tecnológicas, e reportados usando categorias descritivas, que são

geralmente quantitativas. É característica da ciência natural, moderna enfatizar a obtenção deste tipo de conhecimento; e, por causa disso, as suas metodologias envolvem a adoção das estratégias descontextualizadas [EDs], i.e., estratégias que restringem as teorias, que podem ser consideradas em pesquisa (para investigação e avaliação), àquelas que possam representar a ordem causal subjacente dos fenômenos sob investigação (em dissociação dos seus contextos humanos, ecológicos e sociais), e dão prioridade à busca do dados quantitativos. 3

Em contraste às ciências naturais, as ciências sociais não podem dissociar dos contextos dos fenômenos sob investigação, ou evitar o usos de muitas das categorias da vida diária, excluídas das teorias desenvolvidas sob as EDs. Essa é uma das razões para a separação profunda das ciências naturais e sociais, que ainda persiste (ver seção 3).

\section{Fecundidade, versatilidade e utilidade prática}

Sem dúvida EDs são fecundas e versáteis. Fecundas: capacitavam à acumulação de uma quantidade enorme de conhecimento da ordem causal subjacentes aos fenômenos do mundo, tornando possível muitas descobertas de componentes e de leis dessa ordem, além de identificarem possibilidades para ação humana previamente desconhecida. Versáteis: novos tipos de EDs surgem regularmente para tratar fenômenos que não puderam ser entendidos sob EDs antecedentes. Por exemplo: a metodologia newtoniana substitui a metodologia cartesiana para explicar os movimentos dos planetas; as metodologias da mecânica quântica, que permitem o uso de leis probabilísticas, foram introduzidas para tratar fenômenos do domínio subatômico; alguns biólogos teóricos rejeitam metodologias reducionistas em favor de modelos que incorporam a complexidade matemática, para entender fenômenos como o desenvolvimento dos organismos.

$\mathrm{O}$ alcance das EDs continua a se expandir, aparentemente sem nenhum limite em vista. Entretanto, a ausência de um limite neste sentido não implica que (em longo prazo) as EDs serão capazes de fornecer compreensão de todos os fenômenos (LACEY, 2009). As EDs

\footnotetext{
${ }^{3}$ Introduzi em Lacey (1999) a noção de estratégia (que impõe restrições sobre as teorias consideradas para investigação e avaliação num projeto de pesquisa, e seleciona os tipos de dados a procurar e reportar e as categorias a usar na reportagem deles); ver também Lacey (2008a; 2010). O que agora denomino 'estratégias descontextualizadas', denominei nestes livros 'estratégias materialistas'.
} 
NORUS, v. 1, n. 2 Jan -Jun 2014.

faltam recursos necessários para pesquisar certos fenômenos - aqueles essencialmente ligadas os seus contextos, tais como: mudança social, agroecossistemas sustentáveis, e ações humanas, inclusive das atividades da própria pesquisa científica. Mas, algumas vezes o conhecimento desses fenômenos pode ser obtido utilizando estratégias não redutíveis às EDs - estratégias sensitivas a contexto [ECs] - e confirmado à luz dos mesmos critérios empregados sob EDs (LACEY, 2014). Isso é fundamental para as propostas que vou introduzir, concernentes às responsabilidades dos cientistas - acesso a conhecimento, confirmado sob ECs é necessário para os cientistas exercerem as suas responsabilidades (LACEY, 2011a).

Além de ser fecundas e versáteis, as EDs geralmente são avaliadas como úteis. $\mathrm{O}$ uso delas conduz à obtenção do tipo de conhecimento que seja capaz de informar aplicações e, ao mesmo tempo, de explicar a eficácia delas. O valor dessas aplicações geralmente é assegurado para as perspectivas de valor que acordam valor alto ao exercício de controle sobre objetos naturais, e incorporam valores do progresso tecnológico [VPT]. Os VPT admitem alto valor ético e social ao aumento da capacidade humana para controlar objetos naturais, especialmente como incorporadas em inovações tecnológicas; às inovações que aumentem a penetração da tecnologia, sempre mais intrusa em domínios da vida humana; e à definição de problemas (por exemplo: fome em áreas pobres) em termos que permitam soluções tecnológicas informadas por conhecimento científico (LACEY, 2008a; 2010). Embora provavelmente todo o mundo valoriza algumas aplicações do conhecimento obtido sob EDs (em medicina, comunicações etc), atualmente os VPT tendem ser interpretados à luz da sua incorporação social juntamente com os valores do capital e do mercado (VC\&M).

Disse anteriormente que o empreendimento da ciência moderna e a busca do progresso tecnológico reforçavam-se mutuamente. Ciência e tecnologia podem ser distinguidas. Cada uma delas tem a sua própria esfera de empreendimento. Há uma profusão das tecnologias que não pode ser reduzida à ciência aplicada. Além de mais, nem todo o conhecimento estabelecido sob EDs é aplicável tecnologicamente; e frequentemente pesquisa conduzida sob EDs proceda, como ciência básica, de acordo a agendas determinadas por interesses teóricos, bem como o interesse de aumentar nosso entendimento dos fenômenos do mundo e sua ordem causal subjacente - sem atenção à possibilidade imediata (ou não) ou do aumento das capacidades humanas para controle, ou de aplicações tecnológicas (médicas, militares, agrícolas etc), mas talvez não sem a esperança de que eventualmente (quem sabe quando?) aplicações surgiriam da pesquisa básica. 


\section{Horizonte do controle aumentado versus horizonte do entendimento compreensivo}

Existem relações mutuamente reforçadoras entre adoção de EDs e sustentação das VTP, mas não a subordinação de uma à outra (LACEY, 2008a; 2010). O horizonte de controle expandido "enhanced control" sempre foi presente ao longo da ciência moderna. E (desde Galileu até o século XX, e ainda hoje) a tradição científica tem tendido a interpretar esse horizonte em termos do ideal da neutralidade, que a ciência aplicada, e os poderes humanos para controlar objetos naturais, (em princípio) são capazes de servir todas as perspectivas de valor viáveis equitativamente; i.e., a ciência aplicada não privilegia algumas perspectivas à despesa de outras.4 Ao mesmo tempo, outro horizonte era presente também: o horizonte do conhecimento compreensivo: o de obter entendimento de todos os objetos e fenômenos do universo. Era pensado que a ciência básica traria este horizonte mais perto, de um tipo de pesquisa que obtém sempre maior entendimento da ordem causal subjacente e das leis do mundo. Assim, a ciência moderna desenvolvia-se, com os dois horizontes mantidos em vista, acompanhada, geralmente, por um auto entendimento em que: (i) a ciência básica era considerada de alta importância; (ii) fecundidade e versatilidade eram distinguidas da utilidade (embora talvez não sempre claramente separadas); e (iii) as prioridades da pesquisa científica eram determinadas dentro de instituições que mantinham considerável autonomia em relação às perspectivas de valor servidas por aplicações úteis do conhecimento científico (LACEY \& MARICONDA, 2012; cf. OLIVEIRA, 2011).

O uso de EDs era visto como a chave para levar ambos os horizontes mais perto ao domínio de experiência e valor humana, sem um obscurecer o outro. Mas, quando a pesquisa está conduzida exclusivamente sob EDs, torna-se difícil prevenir que o horizonte do controle expandido obscurecerá o outro horizonte. Esta dificuldade poderia ficar invisíveis para aqueles que enfatizavam que não existam limites à fecundidade e versatilidade das EDs, e pensam - erroneamente (ver 1.2) - que este significa que (em princípio) todos os fenômenos poderiam ser compreendidos sob as EDs. A fonte das dificuldades é que as EDs não possuem os recursos necessários para investigar adequadamente os fenômenos essencialmente ligados

\footnotetext{
${ }^{4}$ Para análise mais detalhada do ideal da neutralidade, e discussão das suas variações, ver Lacey (2010, cap. 1;
} 2013); cf. Oliveira (2008a). 
aos seus contextos (1.2) e, portanto, que é necessário utilizar alguns ECs para investigá-los. É um engano supor que a investigação científica é definida pelo uso das EDs. O que é essencial para uma investigação científica é avaliação empírica dos seus resultados à luz dos critérios padrões para avaliação cognitiva (Lacey, 2012; 2014).

Portanto, o horizonte do entendimento compreensivo não faz sentido separadamente do fato de aceitar o pluralismo metodológico (LACEY, 2008b, 2010, 2014) que incorpora não só a variedade das EDs introduzida historicamente, mas também as ECs apropriadas. Além disso, sem o pluralismo metodológico o horizonte do controle aumentado não pode refletir o ideal da neutralidade - porque a pesquisa, conduzida quase exclusivamente sob EDs não ajusta-se bem para servir àquelas perspectivas de valor, sustentadas em sociedades democráticas, que não incorporam os VTP (LACEY, 2008b; 2013). Sem o pluralismo metodológico, é provável que o horizonte do entendimento compreensivo vá retroceder da nossa vista e permanecer sempre mais obscurecido pelo horizonte do controle aumentado. Mas a tradição científica moderna resistia o pluralismo metodólogo que inclui ECs, e geralmente não questiona a primazia e quase exclusividade das EDs. Então, torna-se difícil, se não impossível, manter os dois horizontes em vista, ao mesmo tempo.

\section{Tecnociência}

Esta situação cria a oportunidade para introduzir uma nova ordem de prioridades, fornecida pela tecnociência - trazendo muito mais perto o horizonte do controle aumentado, de interpretar os VTP em termos da sua interação dialética com VC\&M, e de permitir o horizonte do entendimento compreensivo de retroceder da preeminência na pesquisa atual.

Em tecnociência ${ }^{5}$ a tecnologia mais avançada é usada para produzir instrumentos, objetos experimentais, e novos objetos e estruturas que possibilitam a obtenção do conhecimento de objetos, eventos e estados de coisas em novos domínios, especialmente conhecimento do que podemos fazer e construir, com o objetivo de inovação prática, industrial, médica, etc., além do crescimento econômico e da competição, sempre levados em consideração. Mesmo quando inovação tecnocientífica não é o objetivo imediato dum projeto de pesquisa, produtos e inovações tecnocientíficos são requisitos para conduzir a pesquisa instrumentos para medida, aparelho experimental, e computadores com capacidade grande para analisar dados e simular processos.

\footnotetext{
${ }^{5}$ Nesta seção, sumario matéria em Lacey (2012). Para discussão de vários assuntos ligados à tecnociência ver o
} 'Special Issue' da revista, Scientiae Studia, publicada em julho de 2012. 


\section{Inovação Tecnocientífica e as responsabilidades de cientistas e Cientistas Sociais}

Assim, inovação tecnocientífica pode ser um produto colateral de criar e implementar as condições necessárias para conduzir pesquisa. Com tecnociência, as tendências enfatizadas na ciência tornam-se aquelas que exploram a contribuição tecnológica de e para pesquisa; e, direta ou indiretamente, aumentam nossos poderes para intervir no mundo e controlá-lo, especialmente poderes que prometem servir interesses das VC\&M, mesmo que solapem outros valores (por exemplo, sustentabilidade ou segurança alimentar dos pobres). Este favorece aqueles interesses que ganham do crescimento econômico - e frequentemente também interesses vinculados aos valores tais como liberdade individual, saúde pessoal, e segurança nacional; interesses que tendem de ser imbricados com crescimento econômico. A preeminência da tecnociência fornece força adicional para privilegiar EDs e para não tomar seriamente a proposta do pluralismo metodológico (que contém SCs). Tecnociência é pesquisa conduzida sob EDs; e os objetos investigados, as possibilidades exploradas, e alguns dos métodos específicos utilizados são de interesse especial àqueles que sustentam os VC\&M. Grande parte do financiamento para pesquisa tecnocientífica está motivada por ligações entre pesquisa científica, inovação tecnocientífica; ganhar vantagens econômicas e crescimento econômico.

A tecnociência apresenta uma nova ordem e interpretação de prioridades, mas não um corte com a tradição científica moderna. Na tecnociência, o horizonte do entendimento compreensivo retrocede e fica significativamente obscurecido. Entretanto, nela, a ciência básica continua a ser conduzida sob EDs. Mas, agora, dado que ela não tem a finalidade direta de obter resultados práticos, é vista como tendo menos valor, não só pelas corporações que financiam muita pesquisa, mas também dentro de círculos científicos notáveis, onde obter patentes de produtos da pesquisa tornou-se considerado um critério importante par avaliar a produtividade científica (OLIVEIRA, 2008b; 2009; 2013).

A ciência básica tem menos valor, embora ainda retenha valor: pesquisa tecnocientífica, que visa imediatos resultados práticos, não pode ser conduzida sem a contribuição da ciência básica e a disponibilidade de instrumentos apropriados para a manipulação (ou observação) de materiais. A pesquisa que produziu os transgênicos, por exemplo, tem dependido do conhecimento das estruturas moleculares de genomas de sementes e plantas; curas prometidas a partir da pesquisa sobre células-tronco dependem de entendimento do desenvolvimento das células; nanotecnologia tornou-se possível depois da construção de instrumentos que tornaram possíveis a manipulação de moléculas individuais. 
NORUS, v. 1, n. 2 Jan -Jun 2014.

Consequentemente, a pesquisa tecnocientífica, que envolve investigações teóricas ou a construção de instrumentos novos, embora distante dos interesses imediatos do mercado, seja bem financiada (por agências governamentais e universidades, geralmente não por corporações) - algumas vezes por causa do reconhecimento da sua necessidade para o desenvolvimento de certos tipos de produtos, mas outras vezes só com a expectativa que "um dia no futuro" a pesquisa levará a "pay-off". Seja como for, dentro da nova ordem da tecnociência o valor da pesquisa deriva-se da sua contribuição (seja imediato, seja no futuro) para o fortalecimento das VTP e VC\&M.

\section{RESPONSABILIDADES DOS CIENTISTAS}

Os cientistas incorrem em responsabilidades no curso de conduzir pesquisa, e publicar resultados científicos e resultados esperados. Também, se (quando) participarem em deliberações pertinentes a políticas públicas (por exemplo, sobre inovações tecnocientíficas e a legitimidade das suas implementações), especialmente no que diz respeito a decisões sobre as prioridades de pesquisa, e de juízos sobre as credencias científicas de reivindicações do conhecimento científico (por exemplo, a respeito do aquecimento global) e à credibilidade de suas evidências empírica. A responsabilidade primária dos cientistas é manutenção da integridade da ciência6.

Na perspectiva da tecnociência, o horizonte do entendimento compreensivo tornou-se obscurecido pelo horizonte do controle aumentado (interpretado à luz das VC\&M) - que enfatiza o valor do aumento do conhecimento sobre o que podemos fazer, e de como podemos aumentar poderes para exercer controle sobre objetos, especialmente pela causa de crescimento econômico e outros interesses das grandes corporações. Segue daí que as implementações de inovações tecnocientíficas geralmente tem valor; e parece plausível para fazer a inferência da eficácia de uma inovação à legitimidade da sua implementação normalmente imediata e sem mais atraso - ao menos que pesquisas empíricas (conduzidos sob EDs) demonstrarem a existência de riscos sérios que não possam ser administrados adequadamente, (LACEY, 2008b). Aqui, há acordo geral de que cientistas têm responsabilidade para conduzir suficientes investigações de risco antecedente à implementação de inovações7.

\footnotetext{
${ }^{6}$ Para mais discussão das responsabilidades dos cientistas, ver Lacey (2008b; 2011a; 2014); Oliveira (2008b).

${ }^{7}$ Mas, ver a discussão de análise de risco padrão (abaixo).
} 


\section{Inovação Tecnocientífica e as responsabilidades de cientistas e Cientistas Sociais}

Muitos cientistas, porém, afirmam que não têm outras responsabilidades a respeito dos riscos; isso em parte porque eles estão convencidos da neutralidade da inovação tecnocientífica, ou seja, de que (em princípio), para qualquer perspectiva de valor viável no mundo contemporâneo pode se identificar implementações de inovações eficazes, consideradas desejáveis para aquela perspectiva. Além do mais, de vez em quando, outras atitudes éticas acompanham a inferência da eficácia à legitimidade: que é obrigatório buscar soluções tecnocientíficas para os problemas grandes da sociedade (por exemplo, a fome, destruição ambiental, mudança climática), e lhes dar prioridade; que é irresponsável por em dúvida o potencial ou legitimidade da pesquisa proposta para produzir tais "soluções"; e que é obrigatório (e não apenas legítimo) utilizar inovações tecnocientíficas (LACEY, 2008b).

Explicita ou implicitamente, os VC\&M influenciam a interpretação, ceteris paribus, do que qualifica a inferência da eficácia à legitimidade (LACEY, 2008a). Mas em uma democracia, juízos de legitimidade não podem ser adequadamente baseados apenas nos VC\&M. Então, o juízo de valor - as responsabilidades dos cientistas são desempenhadas quando eles concluem que investigações suficientes e adequadas de risco foram conduzidas precisa ser questionado (LACEY, 2011b). Normalmente, a eficácia é uma condição necessária para a legitimidade da implementação de uma inovação; mas também é necessário levar em conta benefícios (e a sua distribuição), danos esperados (e para quem), riscos, e alternativas (existem alternativas melhores, com benefícios comparáveis ou maiores, e menores riscos?). Na condição que não existem conflitos de interesse, a ciência, conduzida sob EDs é autoritária a respeito de assuntos da eficácia, mas precisamos usar ECs para investigar os outros assuntos, porque não se pode investigá-los adequadamente sem levar em conta o contexto ecológico e social da implementação de uma inovação. Ademais, a consideração desses outros assuntos envolve necessariamente juízos de valor ético e social. 'Risco', p. ex., é um termo com conotações éticas: identificar um efeito potencial como um risco, implica que deve ser evitado ou seu impacto minimizado. Porém, os cientistas, enquanto cientistas, não possuem competência especial para tratar de assuntos de valores, ou para fazer juízos de valor ético ou social (LACEY, 2009; 2014).

\section{Primeira proposta:}

As deliberações, que conduzem à formação de política pública sobre a legitimidade da implementação de uma inovação tecnocientífica, devem ser bem informadas por todo conhecimento científico relevante, disponível e alcançável, a respeito não só com relação à 
NORUS, v. 1, n. 2 Jan -Jun 2014.

sua eficácia, mas também aos seus benefícios e riscos e às alternativas ao seu uso. Os cientistas são responsáveis para assegurar que suficiente pesquisa - utilizando o pluralismo apropriado de estratégias - atualmente será conduzida, para que evidencias sejam obtidas sobre todos estes assuntos e disponibilizados para informar as deliberações.

Para exercer esta responsabilidade, é necessário dar ampla consideração tanto à natureza de objetos tecnocientíficos (Bensaude-Vincent, et al., 2011), quanto às consequências do seu uso nos contextos, sociais e ecológicos, em que estão e estarão atualmente usados.

Objetos tecnocientíficos são objetos físicos, químicos e/ou biológicos produzidos por meio de intervenções técnicas, experimentais e instrumentais, que incorporam conhecimento científico verificado sob EDs, e que são realizações de possibilidades descobertas ou identificadas em pesquisa conduzida sob EDs. São, também, componentes de sistemas sociais e ecológicas, objetos que incorporam os VTP e (o mais deles) os VC\&M (LACEY, 2012). Planta transgênicas, p. ex., são entidades biológicas, produtos de intervenções técnicas nos genomas de seus antepassados; incorporam os VTP; são componentes de agroecossistemas com dimensões mundiais; são objetos comerciais, cujos usos estão restringidos por títulos de diretos de propriedade intelectual, refletindo que elas incorpora os VC\&M. Os transgênicos têm efeitos (e ocasionam riscos) nos seres humanos e sistemas sociais e ecológicas em virtude de todos os tipos de coisa que elas são: biológicos, ecológicos, objetos sociais que incorpora os VTP e os VC\&M (LACEY, 2006a; 2010, parte 2). A natureza dos objetos tecnocientíficos - seus poderes, tendências, origens causais, efeitos nos seres humanos e sistemas sociais e ecológicos - não pode ser compreendida em investigação que não admite espaço para algumas ECs.

Os riscos dos usos dos objetos tecnocientíficos podem ter vários mecanismos, dada a multiplicidade dos tipos de coisa que eles se constituem: mecanismos socioeconômicos e ecológicos, além dos físicos, químicos e biológicos. Testes de risco adequados devem investigar todos estes riscos. Geralmente, porém, o agronegócio e as comissões regulamentares oficiais - p. ex., CTNBio, Comissão Nacional de Biossegurança (discutido em Tait, 2011) - levam em consideração apenas as análises de risco padrão, que são investigações, conduzida sob EDs, de riscos (ao curto prazo) ocasionados por mecanismos físicos, químicos e biológicos, cuja probabilidade de ocorrência pode ser estimada 


\section{Inovação Tecnocientífica e as responsabilidades de cientistas e Cientistas Sociais}

quantitativamente. 8 Nestes análises, os riscos ocasionados por mecanismos socioeconômicos não são investigadas, inclusive de riscos ao longo prazo para o ambiente e para saúde, e riscos sociais, por exemplo, o solapamento das condições necessitadas para tipos de agricultura que contribuem para proteger a segurança alimentar dos muitas comunidades pobres. Testes dos riscos ocasionados por mecanismos socioeconômicos não podem ser conduzidos exclusivamente sob EDs. ECs precisam ser utilizadas para investigar o papel causal do contexto do uso e os efeitos do uso sobre este contexto, e também as possibilidades de alternativas que não sejam baseadas em inovação tecnocientífica - p. ex, agroecologia (Lacey, 2006a: cap. 5; 2010; caps. 7, 8) e tecnologia social (DAGNINO, 2010). Aqui, juízos relevantes devem ser baseados em conhecimento obtido no curso de investigação sistemática e empírica, cujos resultados são avaliados utilizando os critérios cognitivos relevantes para toda ciência. O tipo de pesquisa que possam informar a política pública concernentes aos assuntos de benefícios, riscos e alternativos precisa utilizar pluralismo metodológico, que inclui algumas ECs.

Contrário à perspectiva e ao espírito tecnocientífica, a primeira proposta implica na necessidade de atrasar implementações (mesmo se os testes de risco padrões conduzidos estiverem considerados suficientes) nas situações seguintes: (1) onde conhecimento científico disponível não fornece suporte suficientemente forte para juízos definitivos sobre os efeitos eticamente saliente duma inovação, o significado (ético e social) deles e a probabilidade que ocasionariam dano sério; mas (2) fornece suporte (ou é consistente com) a plausibilidade da hipótese que a inovação pudesse causar dano ou ocasionar risco sério, e talvez irreversível, a seres humanos, arranjos sociais ou natureza. Estes são as condições em que o Princípio de Precaução aplica-se, e propõe atraso das implementações - para permitir tempo adequado para as investigações relevantes (LACEY, 2006b). O Princípio de Precaução interpreta 'risco sério’ na luz de valores tais como justiça social, respeito para o alcance amplo dos direitos humanos, sustentabilidade ambiental, equidade dentro e entre gerações, e democracia participativa - sem subordinar eles aos VC\&M (LACEY, 2006b).

\footnotetext{
${ }^{8}$ Para mais mais conhecimento das análises de risco padrão e crítica da suficiência e adequação dos testes feitos dos transgênicos atualmente usados, ver Lacey (2011b). Em análises de risco padrão, faz parte da definição de 'risco' que a probabilidade de um risco pode ser estimada, e isso envolve uma distinção entre 'risco' e 'incerteza' (para detalhes, ver Jerónimo e Garcia, 2011). Aqui, e em outros escritos (Lacey, 2006a, 2010), uso 'risco' no sentido mais comum que se inclui ambos os 'riscos' e as 'incertezas'.
} 
NORUS, v. 1, n. 2 Jan -Jun 2014.

Segunda proposta:

O Princípio de Precaução deve informa agendas da pesquisa cientifica; portanto, seria irresponsável participar em pesquisa que visa gerar inovações tecnocientíficas, ao menos que pesquisa rigorosa e sistemática também estiver conduzida (i) nos riscos e incertezas ecológicos e sociais de implementar as inovações, tomando em conta as condições socioeconômicos das propostas implementações e as condições atuais do seu uso, e o fato que 'risco' é um termo com conotações éticas, e (ii) que é pertinente para comparar o valor social das inovações com aquele de alternativas possíveis (que não dependam de inovação tecnocientífica).

\section{Terceira proposta:}

Condições para os cientistas agir de acordo às primeiras duas propostas devem ser disponíveis como uma parte normal da conduta das atividades científicas, e não devem ser postas ao lado frente às pressões 'produtivistas'. Os cientistas são responsáveis por agir e assegurar que essas condições se tornem presentes nas suas instituições.

\section{RELEVÂNCIA PARA AS CIÊNCIAS SOCIAIS}

Finalmente, algumas palavras sobre as ciências sociais. Disse que a separação profunda das ciências naturais e sociais só faz sentido, se as EDs forem consideradas essenciais para a metodologia da ciência natural - mas, nesta proposta, as ciências naturais não são suficientes para fornecer compreensão ampla dos objetos do mundo da nossa experiência no mundo da vida (LACEY, 2009). Embora alguns economistas pensem de outra maneira, não faz sentido dissociar os fenômenos sociais dos seus contextos. Especificamente, quais são os objetos tecnocientíficos, e os seus efeitos no mundo da vida, não podem ser compreendidos dentro dos limites das EDs mas só no contexto da trajetória atual do crescimento econômico e as suas consequências, e assim por meio de utilizar um alcance de estratégias complementares tiradas das ciências naturais, ecológicas, psicológicas e sociais (e das várias experiências transdisciplinares).

As ciências naturais e sociais são complementares (LACEY, 2008a, caps. 7, 8; 2010, cap. 3) - com papeis aos diferentes momentos do processo amplamente inclusivo da investigação em andamento e da utilização dos resultados da investigação para informar 
decisões, ações, políticas públicas no mundo da vida. Por exemplo, a pesquisa conduzida sob EDs induziu à produção dos transgênicos, e explica a eficácia deles e identifica os limites desta eficácia; mas, ao momento da aplicação e consideração da legitimidade do uso dos transgênicos, investigações sociais (e outras que utiliza ECs) são necessárias para a investigação de benefícios, riscos e alternativos. Na luz do papel no mundo atual para inovação que contribui para o crescimento econômico, os cientistas sociais têm responsabilidade para contribuir às investigações integrais às três questões (benefícios, riscos e alternativos). E para esclarecer o tipo do controle social necessário para implementação dos projetos baseados no controle dos objetos naturais. E, também, para explorar quais são as condições e conhecimento/tecnologias necessárias para fortalecer práticas que aspiram para um mundo em que relações do controle (social e dos objetos naturais) seriam subordinadas aos valores da sustentabilidade, justiça social e o bem estar de tudo mundo.

Acho que o papel da inovação tecnocientífica que contribui para o crescimento econômico está na raiz 'da crise e emergência de novas dinâmicas sociais' (o subtítulo do programa do III EICS). Para confrontar esta crise, mantenho que a ciência (e tecnologia) precisa ser reorientada para dar prioridade à questão: “Como deve ser conduzida a pesquisa científica (e por quem), e as tecnologias desenvolvidas e administradas, de modo a assegurar que a natureza seja respeitada, que seus poderes regenerativos não sejam mais solapados, e restaurados sempre que possível, e que o bem-estar e os direitos de todos sejam fortalecidos em todo o mundo?" (LACEY, 2008b). Sem a transformação profunda das instituições científicas atuais, e sem reconhecimento da complementaridade das ciências naturais e sociais, esta questão permanecerá marginalizada e sem efeitos significativos na prática da ciência. Como podem as ciências sociais contribuir para lutar na direção desta transformação?

\section{REFERENCIAS}

BENSAUDE-VINCENT, BERNADETTE, SACHA LOEVE, ALFRED NORDMANN, ASTRID SCHWARZ. Matters of interest: The objects of research in science and technoscience. Journal for General Philosophy of Science 42: 365-383.2011.

DAGNINO, RENATO (org.). Tecnologia Social: ferramenta para construir outra sociedade. 2.ed. Campinas, SP: Komedi. 2010.

JERÓNIMO, HELENA \& GARCIA, JOSÉ LUÍS RISKS. Alternative knowledge strategies and democratic legitimacy: the conflict over co-incineration of hazardous industrialwaste in Portugal. Journal of Risk Analysis 14: 951-967. 2011. 
NORUS, v. 1, n. 2 Jan -Jun 2014.

LACEY, HUGH. Is science value free? values and scientific understanding. London: Routledge. 1999.

- A controvérsia sobre os transgênicos: questões científicas e éticas. São Paulo: Editora Idéias e Letras. 2006a.

“O Princípio de Precaução e a autonomia da ciência”. Scientiae Studia 4: 373-392. 2006b.

Valores e atividade científica 1. São Paulo: Associação Filosófica 'Scientiae Studia'/Editora 34. 2008a.

6: $297-327.2008 b$.

. "Ciência, respeito à natureza e bem-estar humano". Scientiae Studia

"O lugar da ciência no mundo dos valores e da experiência humana". Scientiae Studia 7: 681-701. 2009.

Valores e atividade científica 2. São Paulo: Associação Filosófica 'Scientiae Studia'/Editora 34. 2010.

Studia: 487-500. 2011a.

"A imparcialidade e as responsabilidades dos cientistas". Scientiae Prefácio a Márcia M. Tait, Tecnociência e cientistas: cientificismo e controvérsias na política de biossegurança brasileira, p. 13-29. São Paulo: Editora Annablume. 2011b.

. "Reflections on science and technoscience". Scientiae Studia, 10 (Special Issue): 103-128. 2012.

'On the co-unfolding of scientific knowledge and viable values'. In P. Schroeder-Heister, G. Heinzmann, W. Hodges \& P. E. Bours (eds.), Logic, Methodology and Philosophy of Science. Proceedings of the Fourteenth International Congress. London: College Publications. 2014.

“Rehabilitating neutrality". Philosophical Studies 162: 77-83. 2013.

LACEY, HUGH \& MARICONDA, PABLO. "The Eagle and the Starlings: Galileo's argument for the autonomy of science - how pertinent is it today?" Studies in the History and Philosophy of Science 43: 122-131. 2012.

MARICONDA, PABLO R. "O controle da natureza e as origens da dicotomia entre o fato e valor". Scientiae Studia 4: 453-472. 2006.

OLIVEIRA, MARCOS B. De. "Neutralidade da ciência, desencantamento do mundo e controle da natureza". Scientiae Studia 6: 97-116. 2008a.

"A avaliação neoliberal na Universidade e a responsabilidade social dos pesquisadores". Scientiae Studia 6: 379-387. 2008 b. 
Inovação Tecnocientífica e as responsabilidades de cientistas e Cientistas Sociais

"A estratégia dos bônus: três pressupostos e uma consequência: trabalho, educação e saúde”. Scientiae Studia 7: 419-433. 2009.

“Formas de autonomia da ciência”. Scientiae Studia 9: 527-561. 2011.

On the commodification of science: the programmatic dimension. Science \& Education 22: 2463-2483. 2013.

TAIT, MÁRCIA M. Tecnociência e cientistas: cientificismo e controvérsias na política de biossegurança brasileira. São Paulo: Editora Annablume. 2011. 